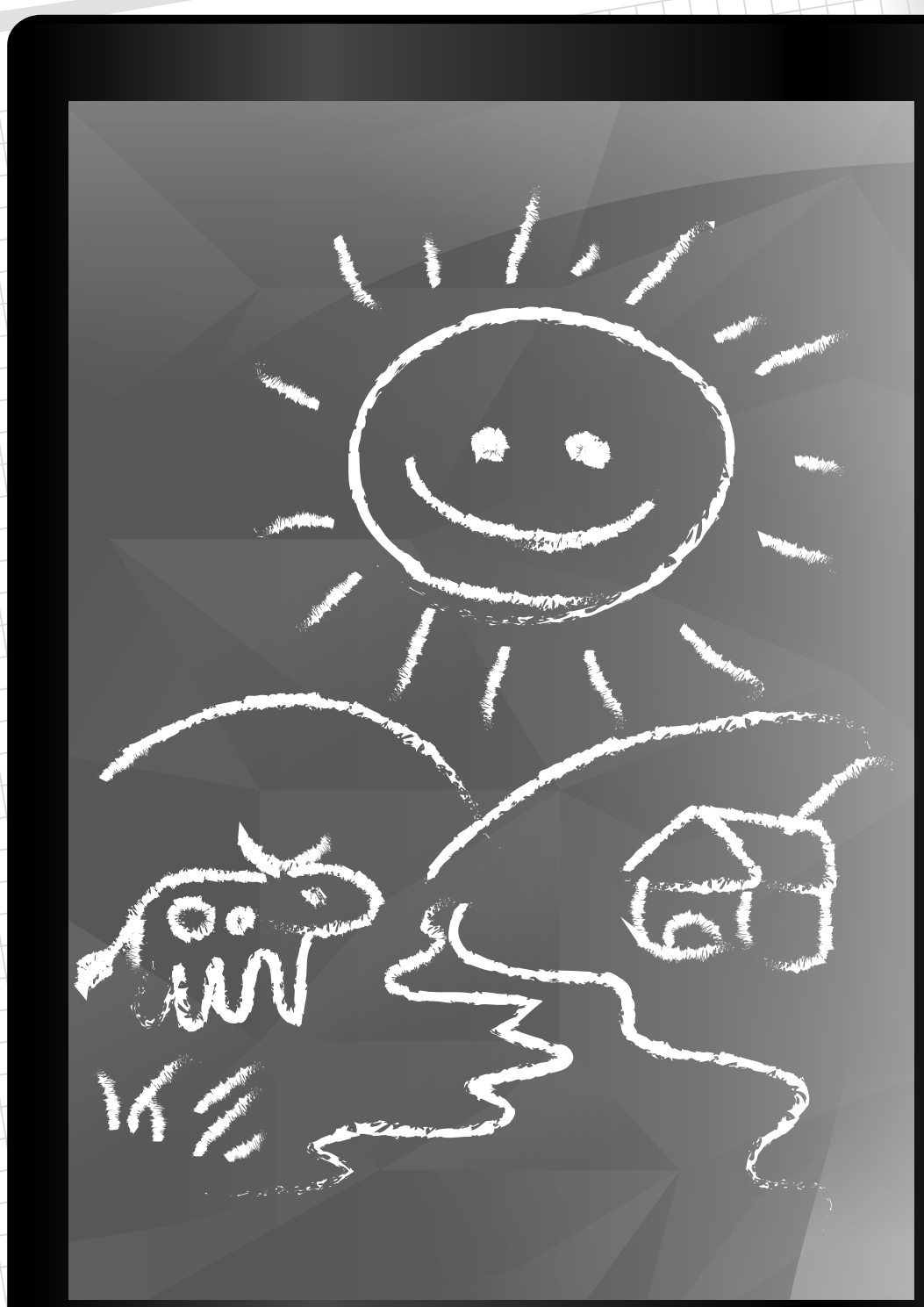

(1)

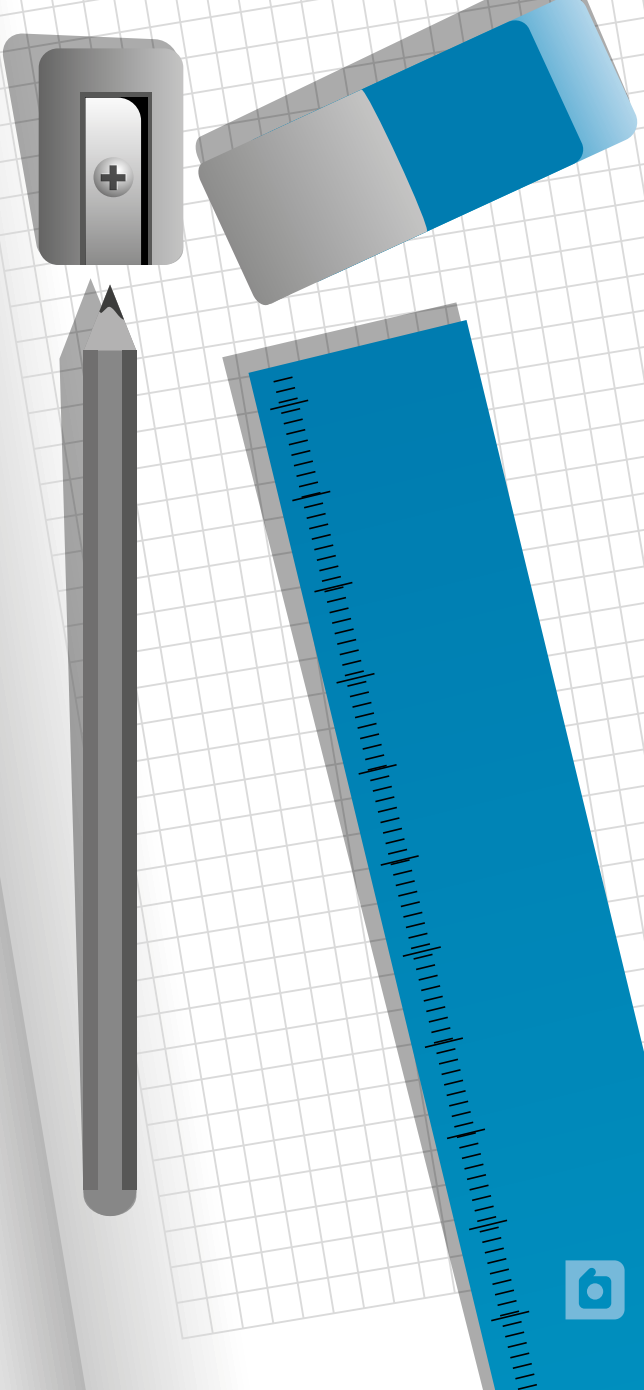

\title{
Entre tabletas, tintas, redes y tecnomediaciones: laberintos de la escuela por explorar
}

Among tablets, inks, networks and tecnomediaciones: labyrinths of school to explore Entre tablets, tintas, redes e tecnomediaciones: labirintos da escola para explorar

1 Texto que hace parte de las primeras reflexiones del proyecto Saberes Tecnomediados de niños, niñas, jóvenes y maestros, desarrollado por el IDEP, en convenio con la Universidad Distrital Francisco José de Caldas.
Ana Brizet Ramírez / anabrizet@gmail.com Fernando Aranguren / fernaranguren@gmail.com Hernán Riveros / hriveros95@gmail.com 
Ana Brizet Ramírez ${ }^{1}$

Fernando Aranguren ${ }^{2}$

Hernán Riveros ${ }^{3}$
2 Doctoranda en Educación, Universidad Pedagógica Nacional. Licenciada en Básica Primaria y Magister en Investigación Social Interdisciplinaria de la Universidad Distrital Francisco José de Caldas. Maestra del Colegio República de Colombia. Pertenece al Grupo de Investigación: Educación y Cultura Política. Línea de Cibercultura y Educación. Docente de la Maestría en Comunicación-Educación de la Universidad Distrital Francisco José de Caldas.

3 Filósofo, Universidad Nacional de Colombia y Magister en Comunicación, Universidad Javeriana. Docente de planta de la Universidad Distrital Francisco José de Caldas; Coordinador Académico de la Maestría en Comunicación-Educación y de la Especialización en Pedagogía de la Comunicación y Medios interactivos. Miembro del Grupo de Investigación Educación, Comunicación y Cultura de la Universidad Distrital Francisco José de Caldas.

4 Magister en comunicación-Educación; especialista en Pedagogía de la comunicación y medios interactivos; licenciado en lingüística y literatura con énfasis en literatura. Docente de la Maestría en comunicación-Educación y de la especialización en desarrollo humano con énfasis en procesos afectivos y creatividad de la Universidad Distrital Francisco José de Caldas. Miembro del grupo de Investigación Educación, Comunicación y Cultura.

\section{Entre tabletas, tintas, redes y tecnomediaciones: laberintos de la escuela por explorar}

\section{Resumen}

Este artículo recoge un avance del proyecto Saberes Tecnomediados de niños, niñas, jóvenes y maestros, desarrollado por el IDEP y la Universidad Distrital Francisco José de Caldas, en el cual se identifican propuestas que desarrollan los maestros del Distrito Capital de distintas localidades, para potenciar en niños, niñas y jóvenes, saberes, narrativas y prácticas que parten de usos y apropiaciones diversas de las tecnologías de la información y la comunicación.

A continuación se relacionan las experiencias seleccionadas, se aborda una primera aproximación descriptiva del contexto escolar, los roles y actores de los escenarios de las propuestas, el lugar desde donde se asumen las tecnologías de la información y la comunicación, así como sus procesos de sostenibilidad, y se da cierre con lo que los autores denominan apuntes para continuar identificando elementos necesarios para el debate educativo y pedagógico.

Palabras clave: tecnologías de la información y la comunicación, saberes sobre tecnología, pedagogía y tecnología.

\section{Among tablets, inks, networks and tecnomediaciones: labyrinths of school to explore}

\section{Summary}

This article includes an advance draft of Knowledge Tecnomediados children, youth and teachers, developed by the IDEP and Francisco José de Caldas District University where teachers develop proposals Capital District of different locations are identified, to enhance in children and young people, knowledge, narratives and practices that are based on various uses and appropriations of information technology and communication. Then the selected experiences are related, a first descriptive approach the school context, roles and actors from the stage of the proposals, the place where the information technology and communication are assumed and their sustainability processes is addressed , and close to what the authors call notes to continue to identify elements necessary for the educational and pedagogical debate occurs.

Keywords: information technology and communication, knowledge of technology, pedagogy and technology.

\section{Entre tablets, tintas, redes e tecnomediaciones: labirintos da escola para explorar}

\section{Resumo}

Este artigo inclui um projecto de antecedência das crianças Conhecimento Tecnomediados, jovens e professores, desenvolvido pelo IDEP e Universidade Francisco José de Caldas Distrito onde os professores desenvolver propostas Capital Distrito de diferentes locais são identificados, para melhorar em crianças e jovens, conhecimentos, narrativas e práticas que são baseadas em vários usos e apropriações de tecnologias de informação e comunicação.

Em seguida, as experiências selecionadas estão relacionadas, uma primeira abordagem descritiva do contexto escolar, papéis e atores da fase das propostas, o local onde a tecnologia da informação e comunicação são assumidos e seus processos de sustentabilidade são os destinatários e próximo ao que os autores chamam de notas para continuar a identificar os elementos necessários para ocorrer o debate educacional e pedagógica.

Palavras-chave: tecnologia da informação e comunicação, o conhecimento da tecnologia, pedagogia e tecnologia. 


\section{Presentación}

El escenario del saber ha cambiado y el campo de la educación no puede estar ajeno a las transformaciones y convergencias tecnológicas, económicas, políticas y culturales de nuestras sociedades contemporáneas. Sea esta la ocasión para replantear sus debates alrededor de las interacciones que se están produciendo con las tecnologías de la información y la comunicación, por parte de niños, niñas, jóvenes y maestros.

El presente artículo observa en una primera aproximación, las relaciones, prácticas, usos y discursos de algunas de las experien- cias seleccionadas en el marco del proyecto Saberes Tecnomediados de niños, niñas, jóvenes y maestros; sin embargo, sabemos que existen muchas otras que están por visibilizarse e intuimos que en ellas se agencian procesos innovadores en el campo de la comunicación-educación. En su conjunto, empezamos a entrever que cobra enorme vigencia el manejo de aparatos, dispositivos y conceptos, como los procesos de inclusión, alfabetización y ciudadanía, desde las tecnomediaciones en el espacio escolar.

\section{Experiencias Tecnomediadas en Bogotá}

En el Distrito Capital, el IDEP, en alianza con la Universidad Distrital Francisco José de Caldas, específicamente, con el Grupo de Investigación Educación, Comunicación y Cultura, realizó dentro del proyecto "Saberes Tecnomediados de niños, niñas, jóvenes y maestros" , una convocatoria pública cuyo objeto se dirigía al reconocimiento de experiencias que desarrollan los maestros y maestras de instituciones educativas, basadas en el uso y apropiación de las tecnologías digitales durante el 2013.

Los criterios que se tuvieron en cuenta para la escogencia de las propuestas fueron:

1. Que evidenciaran algún nivel de apropiación teórica en el campo comunicación-educación o de uso de las TIC en el aula.
2. Que incorporaran tecnologías digitales o análogas, superando la instrumentalidad, y que entraran en diálogo con procesos de pensamiento, alfabetización, ciudadanía y/o creatividad.

3. Que vincularan diferentes grupos y escenarios de la comunidad y del entorno escolar.

4. Que presentaran metodologías inclusivas y propiciaran procesos significativos de aprendizaje.

5. Que manejaran diversas posibilidades de mediación a nivel conceptual y de apropiación de las NTIC.

Con estos elementos, se identificaron 22 propuestas a saber:

\begin{tabular}{|c|c|c|c|c|}
\hline N. & Titulo & Líder & Colegio & Sentido \\
\hline 1. & $\begin{array}{l}\text { Mediación del discurso } \\
\text { infantil por parte de la } \\
\text { televisión: influencia en la } \\
\text { oralidad y adquisición de } \\
\text { habilidades para la lecto - } \\
\text { escritura }\end{array}$ & $\begin{array}{l}\text { - María Jineth Rojas } \\
\text { Vanegas }\end{array}$ & $\begin{array}{l}\text { - Colegio La Victoria } \\
\text { (I.E.D)- Localidad San } \\
\text { Cristóbal }\end{array}$ & $\begin{array}{l}\text { Se caracteriza por indagar la significatividad del } \\
\text { medio televisivo en los niños y niñas de primer } \\
\text { ciclo, con el fin de establecer su incidencia en el } \\
\text { desarrollo de las habilidades comunicativas; para } \\
\text { tal fin, el análisis del discurso es la metodología } \\
\text { apropiada para cuestionar la mediación audiovisual } \\
\text { en la adquisición del código lingüístico en los } \\
\text { estudiantes. }\end{array}$ \\
\hline 2. & Entre -TIC-TAB- aprendo más & - $\quad$ Azucena Parra Soler & $\begin{array}{l}\text { - Colegio Costa Rica } \\
\text { (I.E.D)- Localidad } \\
\text { Fontibón }\end{array}$ & $\begin{array}{l}\text { Asume las TIC como una mediación significativa en } \\
\text { el aprendizaje de una segunda lengua, en la que las } \\
\text { fotografías y los videos se constituyen en fuente } \\
\text { crítica de trabajo colaborativo, para el desarrollo } \\
\text { creativo y participativo de las competencias } \\
\text { lingüísticas y comunicativas en los estudiantes. }\end{array}$ \\
\hline
\end{tabular}




\begin{tabular}{|c|c|c|c|c|}
\hline N. & Titulo & Líder & Colegio & Sentido \\
\hline 3. & $\begin{array}{l}\text { OVA para el desarrollo del } \\
\text { pensamiento científico en } \\
\text { estudiantes de Ciclo I del } \\
\text { Colegio Grancolombiano }\end{array}$ & $\begin{array}{ll}\text { - } \quad \text { Martha Patricia Parra } \\
\text { - } \quad \text { María Claudia Trujillo } \\
\text { - } \quad \text { Stella Soler Villanueva }\end{array}$ & $\begin{array}{l}\text { - Colegio } \\
\text { Grancolombiano } \\
\text { (I.E.D)- Localidad Bosa }\end{array}$ & $\begin{array}{l}\text { Pretende generar procesos de alfabetización digital } \\
\text { crítica en la comunidad educativa a partir de un } \\
\text { OVA, con el fin de trascender tanto en el uso } \\
\text { instrumental y precario de las tecnologías, en el } \\
\text { ciclo inicial, como en sus aprendizajes. }\end{array}$ \\
\hline 4. & $\begin{array}{l}\text { Casos y cuentos de la profe } \\
\text { Brujelia: serie audiovisual }\end{array}$ & $\begin{array}{ll}\text { - } & \text { Danny Andrés } \\
\text { Benavides Portilla } \\
\text { - } \\
\text { Hernando Martínez - } \\
\text { Rector }\end{array}$ & $\begin{array}{l}\text { - } \quad \text { Colegio Nueva } \\
\text { Esperanza (I.E.D)- } \\
\text { Localidad Usme }\end{array}$ & $\begin{array}{l}\text { Promueve la producción de ambientes audiovisuales } \\
\text { con los estudiantes, que los alfabeticen } \\
\text { críticamente y los animan a crear obras para poder } \\
\text { asumir una posición analítica frente a la violencia } \\
\text { y la tramitación de los conflictos escolares. El arte, } \\
\text { la escritura, la fantasía y el abordaje digital, se } \\
\text { constituyen en elementos fundamentales de esta } \\
\text { propuesta, personificada en una bruja docente } \\
\text { que los lleva a generar narrativas en torno a una } \\
\text { convivencia más armónica. }\end{array}$ \\
\hline 5. & Quicagua: entre saberes & $\begin{array}{l}\text { - Mónica Yasmín } \\
\text { Cuineme }\end{array}$ & $\begin{array}{l}\text { - } \quad \text { Colegio La Gaitana } \\
\text { (I.E.D)- Localidad Suba }\end{array}$ & $\begin{array}{l}\text { Se consolida como un proyecto pedagógico en } \\
\text { torno a los saberes que los estudiantes de primer y } \\
\text { segundo ciclo, tienen sobre las riquezas de la tierra } \\
\text { y el ambiente. Se desarrolla desde la huerta escolar } \\
\text { y la convergencia entre tecnologías tradicionales } \\
\text { y digitales. Allí, niños y niñas sistematizan sus } \\
\text { aprendizajes y los socializan en el diálogo entre } \\
\text { pares. }\end{array}$ \\
\hline 6. & Camicreando & $\begin{array}{ll}\text { - } & \text { Doris Rodríguez } \\
\text { - } & \text { Ana Fabiola Cifuentes }\end{array}$ & $\begin{array}{l}\text { - Colegio Ciudadela } \\
\text { Educativa de Bosa } \\
\text { (I.E.D) - Localidad } \\
\text { Bosa }\end{array}$ & $\begin{array}{l}\text { Pretende minimizar las dificultades de aprendizaje } \\
\text { en los niños y niñas entre los } 6 \text { a } 8 \text { años, a partir } \\
\text { del uso y apropiación de los medios interactivos, } \\
\text { en aras de incidir en su formación integral. La } \\
\text { familia ha sido vital para fortalecer la lectoescritura } \\
\text { y el trabajo en valores, desde el ser, el hacer y } \\
\text { el saber. En la página CAMICREANDO.jimdo.com, } \\
\text { recogen la producción de ambientes denominados } \\
\text { Camiestaciones. }\end{array}$ \\
\hline 8. & Cátedra María Cano & $\begin{array}{ll}\text { - } & \text { Martha Ligia Gil } \\
\text { - } & \text { María Andrea Ruiz } \\
\text { - } & \text { Leidy Celis } \\
& \text { Edinson Albeiro } \\
\text { - } & \text { Jenzán } \\
\text { - } & \text { German Arévalo Guzmán }\end{array}$ & $\begin{array}{l}\text { - Colegio Maria Cano } \\
\text { (I.E.D)- Localidad } \\
\text { Rafael Uribe Uribe }\end{array}$ & $\begin{array}{l}\text { Asume las tecnologías digitales como una } \\
\text { oportunidad para fortalecer la formación ciudadana } \\
\text { desde la Cátedra María Cano. Es así, como se } \\
\text { centra en el trabajo pedagógico de los valores, } \\
\text { la recuperación de la memoria y el sentido de } \\
\text { pertenencia, el acompañamiento familiar, la } \\
\text { creación de conciencia ambiental y el uso crítico de } \\
\text { las redes sociales. }\end{array}$ \\
\hline 9. & $\begin{array}{l}\text { Diseño, elaboración e } \\
\text { implementación de un AVA } \\
\text { para estudiantes del Colegio } \\
\text { Alfonso López Michelsen }\end{array}$ & - $\quad$ Segundo Fúquen & $\begin{array}{l}\text { - Colegio Alfonso López } \\
\text { Michelsen (I.E.D)- } \\
\text { Localidad Bosa }\end{array}$ & $\begin{array}{l}\text { Propone desarrollar procesos de pensamiento } \\
\text { tecnológico en estudiantes de educación básica } \\
\text { primaria, que desde el enfoque de la interdisciplina, } \\
\text { incidan en sus aprendizajes. De esta manera, } \\
\text { considera que la articulación entre la exploración } \\
\text { del diseño de artefactos y las tecnologías } \\
\text { digitales, son el camino propicio para potenciar } \\
\text { modos interactivos para aprender significativa y } \\
\text { colaborativamente con la familia. }\end{array}$ \\
\hline
\end{tabular}




\begin{tabular}{|c|c|c|c|c|}
\hline N. & Titulo & Líder & Colegio & Sentido \\
\hline 10. & Tecnobot & $\begin{array}{ll}\text { - } & \text { Diana Carolina Prada } \\
\text { - } & \text { Alura Cely } \\
\text { - } & \text { Lina Soraya Herrera } \\
& \text { Carmen Natalia Pérez }\end{array}$ & $\begin{array}{l}\text { - Colegio María Cano } \\
\text { (I.E.D) - Localidad } \\
\text { Rafael Uribe Uribe }\end{array}$ & $\begin{array}{l}\text { Plantea la necesidad de generar espacios } \\
\text { interdisciplinarios a partir del reconocimiento de los } \\
\text { múltiples saberes y contextos de vida de los estudiantes. } \\
\text { Para ello, promueve el desarrollo de las competencias } \\
\text { y habilidades comunicativas, empresariales, artísticas } \\
\text { y tecnológicas, las cuales se materializan en el diseño, } \\
\text { implementación y socialización de proyectos que } \\
\text { problematizan su realidad. }\end{array}$ \\
\hline 11. & $\begin{array}{l}\text { Red Social Académica Tic, } \\
\text { Tic ¿Puedo Entrar? }\end{array}$ & $\begin{array}{ll}\text { - } & \text { Nelcy Laverde } \\
\text { - } & \text { María Isabel Parra } \\
\quad \text { Nancy López }\end{array}$ & $\begin{array}{l}\text { Colegio Instituto } \\
\text { Técnico Industrial } \\
\text { Francisco José de } \\
\text { Caldas (I.E.D) - } \\
\text { Localidad Engativá }\end{array}$ & $\begin{array}{l}\text { Asume las tecnologías de la información y la } \\
\text { comunicación, como el camino para generar } \\
\text { ambientes de aprendizaje colaborativo en toda la } \\
\text { comunidad educativa. Promueve la cultura digital al } \\
\text { servicio de la pedagogía, para agenciar la formación } \\
\text { como ciudadanos en estudiantes, maestros y padres } \\
\text { y madres de familia, a partir de redes sociales desde } \\
\text { las que se moviliza la inteligencia colectiva y la } \\
\text { democratización del conocimiento. }\end{array}$ \\
\hline 12. & RED Escuela - Familia & $\begin{array}{ll}\text { - } & \text { Luis Eduardo Pulido } \\
\text { - } & \text { Rosa Milena Romero }\end{array}$ & $\begin{array}{l}\text { - Colegio Alfredo Iriarte } \\
\text { (I.E.D)- Localidad } \\
\text { Rafael Uribe Uribe }\end{array}$ & $\begin{array}{l}\text { Se apropia de los medios como parte de los } \\
\text { procesos de aprendizaje desde la transversalidad } \\
\text { curricular. Destaca la participación de los padres y } \\
\text { de los estudiantes en la construcción de ambientes } \\
\text { colaborativos y dialógicos, con un enfoque social y } \\
\text { ciudadano de las tecnologías. Usa blogs y Edmodo, } \\
\text { para vincular la familia, la escuela y la ciudad, a sus } \\
\text { proyectos de vida. }\end{array}$ \\
\hline 13. & $\begin{array}{l}\text { Desarrollo de habilidades } \\
\text { comunicativas en el área de } \\
\text { ciencias naturales con niños } \\
\text { de primaria, a través del uso } \\
\text { de blog }\end{array}$ & $\begin{array}{l}\text { - Marisol Roncancio } \\
\text { López }\end{array}$ & $\begin{array}{l}\text { - Colegio La Aurora } \\
\text { (I.E.D) - Localidad } \\
\text { Usme }\end{array}$ & $\begin{array}{l}\text { Integra el escenario del pensamiento científico y el } \\
\text { campo de la comunicación, con el fin de propiciar } \\
\text { mediaciones interdisciplinares que incidan en los } \\
\text { procesos de aprendizaje. En ella, se promueven } \\
\text { estrategias de lecto-escritura de las situaciones } \\
\text { del contexto, problematizadas, sistematizadas y } \\
\text { socializadas, a partir de ejercicios de investigación } \\
\text { de los estudiantes, quienes son acompañados por } \\
\text { sus familias. }\end{array}$ \\
\hline 14. & Aulas sin Fronteras & $\begin{array}{ll}\text { - } & \text { Juan Carlos Torres } \\
\text { - } & \text { Marisol Espinosa } \\
& \text { José Eduardo } \\
\text { Rodríguez }\end{array}$ & $\begin{array}{l}\text { - Colegio Cedid Ciudad } \\
\text { Bolívar (I.E.D)- } \\
\text { Localidad Ciudad } \\
\text { Bolivar }\end{array}$ & $\begin{array}{l}\text { Es una experiencia de educación inclusiva, que } \\
\text { apoya los proyectos de vida de jóvenes y adultos } \\
\text { entre los } 13 \text { y los } 60 \text { años, los fines de semana, que } \\
\text { se encuentran en situaciones de riesgo psicosocial. } \\
\text { Desarrollan un proyecto educativo pertinente a } \\
\text { las necesidades de la población, a través de blogs, } \\
\text { redes sociales, periódicos y canales interactivos, } \\
\text { agenciando procesos de aprendizaje colaborativo } \\
\text { basados en problemas, con un enfoque social y } \\
\text { ciudadano de alfabetización en TIC. }\end{array}$ \\
\hline 15. & Tecnomaravillas & - $\quad$ Armando Ramirez Pérez & $\begin{array}{l}\text { - Colegio Tom Adams } \\
\text { (I.E.D)- Localidad } \\
\text { Kennedy }\end{array}$ & $\begin{array}{l}\text { Anima a los estudiantes de básica primaria al } \\
\text { desarrollo de sus procesos de aprendizaje, desde la } \\
\text { lúdica del diseño tecnológico de juguetes y el uso } \\
\text { de las tecnologías digitales como los blogs. En este } \\
\text { sentido, potencia las capacidades creativas y críticas } \\
\text { de los niños y niñas, donde la familia se involucra } \\
\text { para acompañar la elaboración de los artefactos. }\end{array}$ \\
\hline
\end{tabular}




\begin{tabular}{|c|c|c|c|c|}
\hline N. & Titulo & Líder & Colegio & Sentido \\
\hline 16. & $\begin{array}{l}\text { El blog como herramienta } \\
\text { educativa en el Colegio } 20 \\
\text { de julio }\end{array}$ & $\begin{array}{l}\text { - William Humberto } \\
\text { Camacho Garzón }\end{array}$ & $\begin{array}{l}\text { - Colegio Veinte De Julio } \\
\text { (I.E.D) - Localidad San } \\
\text { Cristóbal }\end{array}$ & $\begin{array}{l}\text { Promueve el uso del blog, como pretexto para } \\
\text { el desarrollo de habilidades comunicativas y de } \\
\text { pensamiento en los estudiantes de los diferentes } \\
\text { ciclos, que los animen a participar críticamente y a } \\
\text { ser protagonistas de sus procesos de aprendizaje. }\end{array}$ \\
\hline 17. & Herramientas BIOTEC & - Dora Ocampo Rozo & $\begin{array}{l}\text { Colegio Republica De } \\
\text { Colombia (I.E.D) - } \\
\text { Localidad Engativá }\end{array}$ & $\begin{array}{l}\text { Potencia el pensamiento científico y tecnológico } \\
\text { desde el uso y apropiación de las tecnologías } \\
\text { digitales, las cuales se ponen en diálogo con } \\
\text { situaciones reales que son problematizadas } \\
\text { y sistematizadas de forma colaborativa en } \\
\text { los diferentes ciclos. Incentiva semilleros } \\
\text { de investigación que asumen la exploración } \\
\text { plurisensorial como el camino para agenciar lecturas } \\
\text { y escrituras críticas de la realidad ambiental, en } \\
\text { bitácoras o diarios de campo, blogs, plataformas } \\
\text { virtuales, entre otras estrategias. }\end{array}$ \\
\hline 18. & Click Travel & $\begin{array}{ll}\text { - } \quad \text { Paola Lucia Ortiz Mora } \\
\text { - }\end{array}$ & $\begin{array}{l}\text { - Colegio Alfonso López } \\
\text { Michelsen (I.E.D) } \\
\text { Colegio José Francisco } \\
\text { Socarrás (I.E.D- } \\
\text { Localidad Bosa }\end{array}$ & $\begin{array}{l}\text { Destaca la inclusión de herramientas como video } \\
\text { juegos, blogs y diversos repertorios digitales en los } \\
\text { procesos de aprendizaje, para lograr el desarrollo de } \\
\text { habilidades cognitivas. Potencia la interactividad } \\
\text { y la interdisciplina desde la lúdica digital, la } \\
\text { vinculación de la familia y el uso crítico de medios, } \\
\text { como ambientes comunicativos de enseñanza- } \\
\text { aprendizaje. }\end{array}$ \\
\hline 19. & Medios e ILE (EFL) & - Iván Potier Hurtado & $\begin{array}{l}\text { - Colegio Enrique Olaya } \\
\text { Herrera (I.E.D)- } \\
\text { Localidad Rafael Uribe } \\
\text { Uribe }\end{array}$ & $\begin{array}{l}\text { Plantea como las TIC se constituyen como una } \\
\text { mediación para el aprendizaje del inglés, donde el } \\
\text { docente cumple un rol determinante en la creación } \\
\text { de ambientes formativos. En este sentido, e-mails, } \\
\text { fotografías, videos, blogs, canales, magazines, entre } \\
\text { otros recursos, son estrategias para el desarrollo } \\
\text { de pensamiento comunicativo, el aprendizaje } \\
\text { colaborativo y la interacción de la comunidad. }\end{array}$ \\
\hline 20. & Plan de actuación en TIC & $\begin{array}{l}\text { - Gustavo Adolfo Duarte } \\
\text { Urueña }\end{array}$ & $\begin{array}{l}\text { - Colegio José Acevedo } \\
\text { y Gómez (I.E.D) - } \\
\text { Localidad San Cristóbal }\end{array}$ & $\begin{array}{l}\text { Propicia en estudiantes de grado décimo y once, el } \\
\text { reconocimiento de las tecnologías desde el enfoque } \\
\text { de alfabetización social, crítica y democrática, } \\
\text { trascendiendo su uso instrumental. Con esta } \\
\text { perspectiva agencia procesos de aprendizaje sobre } \\
\text { el lenguaje de etiquetas HTML, y la creación de } \\
\text { aplicaciones para móviles bajo el sistema operativo } \\
\text { Android. }\end{array}$ \\
\hline 21. & $\begin{array}{l}\text { Las TIC en el proceso de } \\
\text { evaluación y fortalecimiento } \\
\text { de saberes y competencias } \\
\text { en química }\end{array}$ & $\begin{array}{l}\text { - Javier Ignacio Muñoz } \\
\text { Martínez }\end{array}$ & $\begin{array}{l}\text { - Colegio Alfredo Iriarte } \\
\text { (I.E.D) - Localidad } \\
\text { Rafael Uribe Uribe }\end{array}$ & $\begin{array}{l}\text { Propone usar las tecnologías como marcos de } \\
\text { apoyo para el fortalecimiento de los saberes y las } \\
\text { competencias científicas, específicamente de la } \\
\text { química. Las dinámicas que allí se promueven, } \\
\text { potencian apuestas didácticas y evaluativas en las } \\
\text { que los estudiantes asumen un rol protagónico en } \\
\text { sus procesos de aprendizaje. }\end{array}$ \\
\hline
\end{tabular}




\begin{tabular}{|l|l|l|l|}
\hline \multicolumn{2}{|l|}{ Titulo } & \multicolumn{2}{c|}{ Colegio } \\
22. & $\begin{array}{l}\text { Saberes tecnomediados } \\
\text { y aprendizajes formales } \\
\text { e informales en niños de } \\
\text { básica primaria }\end{array}$ & $\begin{array}{l}\text { July Maricela Sepúlveda } \\
\text { Sánchez }\end{array}$ & $\begin{array}{l}\text { Observa la integración de los escenarios online/ } \\
\text { offline en que habitan niños y niñas, como espacios } \\
\text { para indagar por las representaciones sociales sobre } \\
\text { la familia y la infancia, que se constituyen a través de } \\
\text { los ecosistemas comunicativos en que interactúan con } \\
\text { la televisión, el internet, la calle y los ambientes de } \\
\text { socialización primarios. }\end{array}$ \\
\hline $\begin{array}{l}\text { (I.E.D) - Localidad } \\
\text { Rafael Uribe Uribe }\end{array}$
\end{tabular}

Tabla 1. Trabajos seleccionados en el marco de la convocatoria pública del proyecto "Saberes Tecnomediados de niños, niñas, jóvenes y maestros"

Cabe mencionar que luego de seleccionar los trabajos, cada equipo gestor desarrolló una ficha de ampliación para detallar la situación originaria de la experiencia, sus estrategias principales, los referentes fundamentales, así como una descripción de su significado. Posteriormente, se concretó un espacio de conversación para poder afinar la mirada, teniendo en cuenta los siguientes aspectos: nivel teórico, concepciones de educación, pedagogía y didáctica, lugar que le otorga a las TIC, perfiles y roles de los actores, contexto escolar e impacto y formas de sostenibilidad y agenciamiento.

En este momento, se está llevando a cabo un ejercicio inicial de socialización y retroalimentación de la primera versión diagnóstica del estado en que se encuentran los proyectos, desde el que se espera nutrir la perspectiva de análisis para así poder dar paso a la construcción del Co-laboratorio de Experiencias Tecnomediadas.

\section{Contextos escolar, roles y actores}

En su conjunto, las propuestas descritas parten de las necesidades de su contexto, allí tienen lugar, tanto los entornos familiares, personales, barriales y locales, como el aula. El contexto escolar se asume a partir del entramado de las vivencias que tienen los estudiantes en el marco de sus ambientes de vida, ya que éstos influyen estructuralmente en sus costumbres, modos de ser y demandas de aprendizaje.

Es así como estas propuestas se han originado para abordar situaciones psicosociales que rodean a los alumnos y los ponen en situación de alto riesgo de deserción educativa, pero esta misma situación permite que las propuestas se conviertan también en espacios que admiten el empoderamientos de las y los alumnos como ciudadanos en el territorio de la ciudad. Para ello, las tecnologías digitales han permitido, no sólo a los autores de las propuestas, sino a la comunidad, otras formas de relación social, educativa y cultual.

De la misma manera, el fortalecimiento de los aprendizajes académicos y convivenciales se convierte en otra de las premisas para concebir y actuar en torno a las tecnologías, más allá de su sentido instrumental, buscando esencialmente hacer más eficaz la enseñanza. Las entrevistas realizadas con los maestros gestores dan cuenta que más que una herramienta, las tecnologías son percibidas como:

\begin{tabular}{|l|l|}
\hline Tendencia & Categorías \\
\hline \multirow{3}{*}{ TIC como mediación } & Con la cultura \\
\cline { 2 - 2 } & En el aprendizaje \\
\cline { 2 - 2 } & Como mediación comunicativa \\
\cline { 2 - 2 } & Con la cultura, los otros y el conocimiento \\
\cline { 2 - 2 } & Con la cultura extraescolar y con los otros, pero no con la escuela \\
\hline
\end{tabular}




\begin{tabular}{|l|l|}
\hline Tendencia & Categorías \\
\hline TIC como herramienta & En el aula \\
\hline \multirow{2}{*}{ TIC en la gestión } & De contenidos \\
\cline { 2 - 2 } & De información y de procesos administrativos \\
\hline TIC desde la convergencia & Entre tradicionales y nuevas tecnologías \\
\hline TIC como escenario de interacción curricular & Transversalidad e interdisciplinar \\
\hline Uso y apropiación crítica de medios & Alfabetización digital \\
\hline
\end{tabular}

Tabla 2. Relación de la forma en que los maestros gestores perciben las TIC

No obstante, estas categorías, que emergen de las prácticas, no son conscientes y por lo tanto se manejan intuitivamente. Los encuentros de socialización y retroalimentación que están por darse, pretenden llenarlas de contenido y generar un diálogo de saberes desde las realidades particulares para que, de esta forma, sea posible comprender que en la actualidad las interacciones con las tecnologías electrónicas nos muestran otras tecnicidades, aprendizajes y mediaciones de la experiencia humana (Martín-Barbero, 1999; Buckingham, 2002, 2008; Rueda, 2012).

Los encuentros que se realizarán presentarán las distintas perspectivas para abordar la tecnología, teniendo en cuenta elementos como el afianzamiento de los procesos de escritura, la convergencia entre viejos y nuevos medios, la alfabetización crítica, la producción de obras mediáticas basadas en la interdisciplina, el desarrollo de procesos de pensamiento, el diseño de artefactos y juguetes, la discusión en cuanto a las potencialidades de las tecnologías digitales, el software libre y el hacking, así como los asuntos de amenazas y seguridad en las redes sociales. Todo ello a partir del trabajo con Pc, prensa, periódico mural y escolar, celulares, tablets, Tv, radio, cámaras, fotografías, videos, blogs, plataformas virtuales, libros, redes, revistas científicas, software, páginas Web, aulas y canales virtuales.

Por otra parte, cabe destacar dentro de los proyectos, que son los niños, niñas, jóvenes, familias y docentes, los actores que ha- cen que éstos cobren vida. En efecto, las narrativas que concurren en los trabajos manifiestan diversos roles, usos, prácticas e interacciones que están incidiendo en los procesos de socialización y acercamiento al saber de las comunidades, lo cual se nota en el hecho de que un factor común de las propuestas es su interés por atacar amplios niveles de exclusión social y falta de oportunidades económicas, educativas y culturales.

Por ejemplo, la mayoría de las propuestas mencionan que una intención de partida es la inclusión de personas en situación de desplazamiento, con NEE, desvinculadas del conflicto, madres violentadas cabezas de hogar, población en situación de pobreza extrema, niños abandonados, jóvenes en circunstancias psicosociales de alto riesgo, adultos mayores analfabetos, baja comunicación y violencia intrafamiliar, entre otras.

Al mismo tiempo, vale la pena destacar que varios de los estudios seleccionados articulan intereses, vínculos, y saberes de los estudiantes en sus desarrollos a partir de dinámicas colaborativas; aunque, en algunos se mantiene la figura del maestro como el proponente de las estrategias, lo cual da pie a hallar otras formas de construcción didáctica y pedagógica basadas en la corresponsabilidad y en el compartir, así como otros usos y apropiaciones que han de surgir en el diálogo con las nuevas generaciones, para lograr mayor significatividad y articulación a sus experiencias de vida.

\section{Las tecnologías, entre los lugares que adquieren en el aula}

En las apuestas de los docentes se destaca la inquietud y la consolidación de una noción de alfabetización digital que se dirige al uso crítico y creativo de los medios, que promueve modos más colaborativos y participativos para acceder al conocimiento. Los diálogos con los maestros muestran que la principal preocupación no ha sido teorizar ni conceptuar, a nivel pedagógico, las implicaciones de las tecnologías en los procesos de formación de los estudian- 


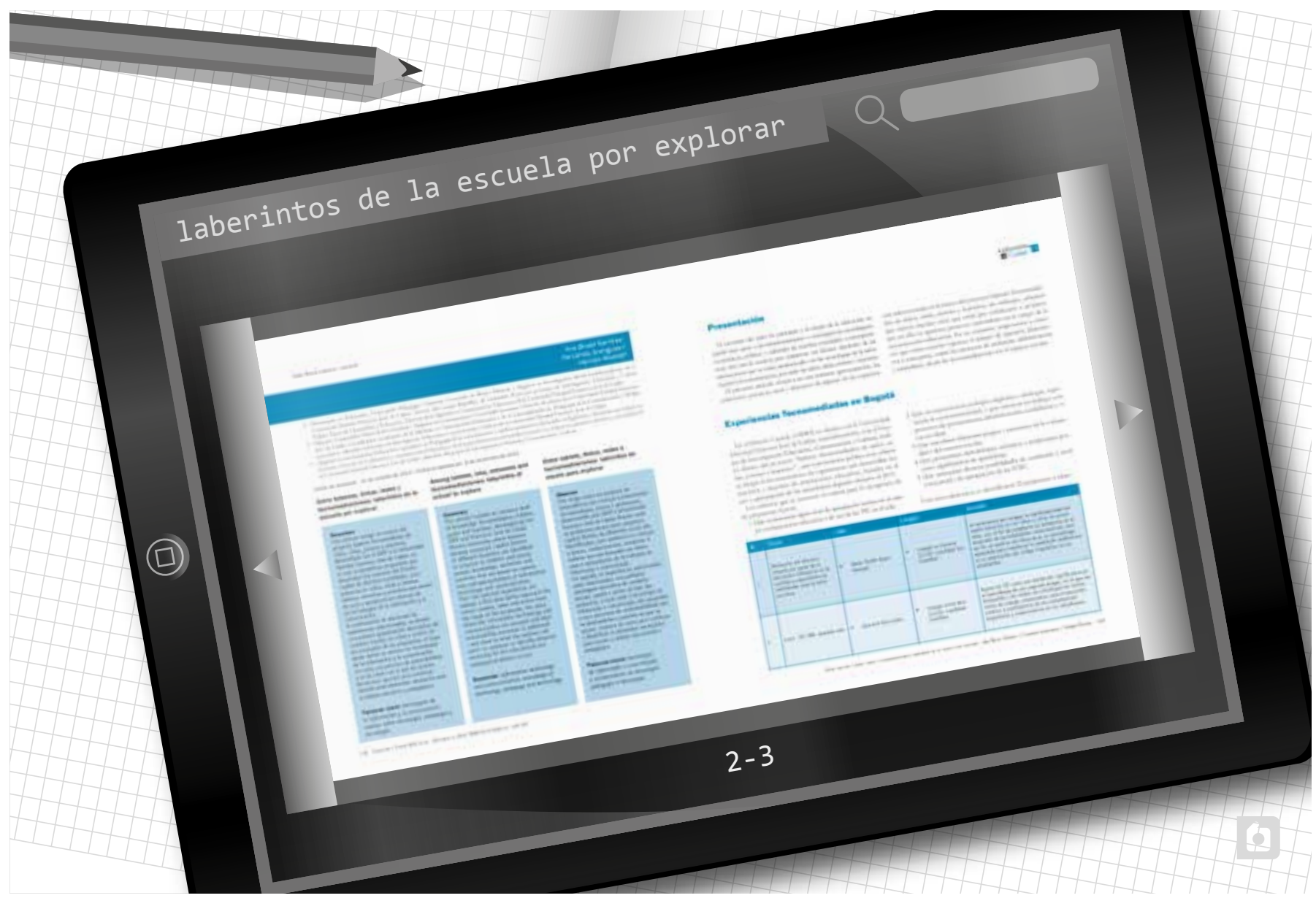

tes, sino el maniobrar con estos dispositivos para cambiar las rutinas en el aula. En un amplio porcentaje, los proyectos notan que existe una desconexión entre la cultura escolar y el mundo real de vida de los niños, niñas y jóvenes, hecho que amplía la brecha entre maestros y estudiantes, puesto que la escuela no reconoce el valor simbólico de las vivencias relacionadas con las nuevas tecnologías.

Los descubrimientos desde la práctica de los gestores de las propuestas, evidencian que aunque no se encuentran formuladas totalmente, sus intencionalidades giran en torno a las posibilidades de transformación de las dinámicas educativas, así como también de la innovación en lo relacionado con la didáctica de las disciplinas; conciben las tecnologías como un escenario alterno a lo tradicional, útil para promover estrategias y talleres en los que con el uso de los medios digitales, se renueven tanto la enseñanza como el aprendizaje.

En este marco, es notable la preocupación esencial por un cambio permanente en el paradigma de escuela, un giro hacia un escenario dialógico y en permanente construcción, en el cual se comprenda efectivamente el conocimiento como un ejercicio interactivo en el que el papel del estudiante es el de protagonista de los nuevos espacios de construcción de sentido; mientras que el maestro funciona como facilitador del mismo a partir de las prácticas mediadas por las tecnologías. Los maestros expresan que los aparatos no son el eje fundamental, ni son determinantes de las acciones; su importancia radica en lo que se puede hacer con ellos, y es lo que genera cambios en las visiones de mundo de las comunidades. 


\section{Procesos de sostenibilidad y agenciamiento de las experiencias}

Uno de los aspectos claves para asegurar el impacto y trascendencia de procesos y proyectos académicos e investigativos como los que se desarrollan a través de este tipo de experiencias educativas innovadoras, tiene que ver con la permanencia de los mismos y su fortalecimiento institucional y operativo, además está también relacionado con su apropiación recurrente y creativa por parte de los actores involucrados. Para ello, es preciso insistir en dos cuestiones determinantes en el logro de estas metas: la sostenibilidad y el agenciamiento.

Se trata de dos dimensiones estructurantes de las políticas, programas e iniciativas que buscan orientar el desarrollo y crecimiento de las actividades humanas, con miras a lograr un mayor y más eficaz impacto en términos de rentabilidad social y cultural y de mejor calidad de vida para la población. Es una visión que, en términos de Nussbaum (2012), aborda la prospectiva de los procesos socioculturales y educativos con base en el fortalecimiento de las capacidades humanas, individuales y colectivas, para que los maestros se asuman y se conviertan en actores de sus propias iniciativas, en protagonistas de los proyectos, que sólo por la apropiación dinámica y proactiva de los mismos resulta factible su materialización.

Lo anterior conlleva un aprendizaje implícito: nada contribuye tanto, de modo tan poderoso y útil, para el propó-

\section{Apuntes para continuar con el debate}

Esta es una primera aproximación, en la que logramos identificar la potencia de reorientar las prácticas pedagógicas a partir de los escenarios que se consolidan alrededor de la técnica y la tecnología, pues allí están germinando procesos amplios de transversalidad curricular que le apuestan a la alfabetización digital, a la vida comunitaria, a la generación de aprendizajes expandidos, y a un uso creativo de las tecnologías, que se revierte en la formación de las nuevas generaciones. Sin embargo, consideramos que este es sólo el punto de partida, pues la discusión está por darse.

En un primer momento, el reconocimiento de los proyectos permite dinámicas de comprensión alrededor de las interacciones y saberes que los maestros promueven con el uso y apropia- sito de transformar positivamente las condiciones iniciales que rodean y determinan un fenómeno o una experiencia, como el propiciar el crecimiento de los directos interesados en tanto sujetos capaces de tomar las decisiones y acciones que lo hagan posible, que garanticen su realización, y no de cualquier forma, sino con arreglo a los parámetros de mayor exigencia. De eso se trata, en consecuencia, el referir la sostenibilidad y el agenciamiento como dimensiones inherentes a los procesos de la experiencia humana. Es la estrategia adecuada para elevar los alcances y la trascendencia moral y social de nuestras acciones productivas y significativas.

$\mathrm{Al}$ abordar lo concerniente a su sostenibilidad y agenciamiento, estamos preguntando por lo que contribuye a fortalecer la experiencia en sí misma, a multiplicarla educativa y pedagógicamente. Algunos de los interrogantes para identificar estos elementos tienen que ver con: la memoria de ejecución y su vínculo con el ejercicio profesional; los factores teórico-prácticos de materialización; las limitaciones y los capitales simbólicos y culturales de los actores, entre otros. Indagar por las condiciones de posibilidad que permiten que una experiencia surja y se sostenga en el tiempo de una forma dinámica, adaptándose a los cambios sociales, políticos y culturales, requiere de un énfasis en el encuadre analítico, para obtener de las experiencias un conocimiento más fino, susceptible de ser refrendado en la práctica misma.

ción de los dispositivos digitales, las cuales dan cuenta, por un lado, del aire de renovación de sus prácticas, con actitudes y disposiciones que se mantienen abiertas y expectantes ante los retos de la sociedad; y por otro lado, evidencian cómo los maestros autogestionan ejercicios de cualificación de su formación docente, actividades que se nutren de los contextos de la escuela para agenciar procesos de aprendizaje colaborativos y situados en las necesidades de las comunidades, así como en sus potencialidades de interacción con múltiples mediaciones de las tecnologías de la información y la comunicación.

En este momento, se están empezando a consolidar espacios de discusión con los actores de las experiencias, para promover 
un diálogo de saberes, prácticas y aprendizajes permanentes desde donde se logren consolidar estrategias de retroalimentación académica, epistemológica y pedagógica, así como de gestión de los proyectos tecnológicos y digitales que emprenden los maestros y los estudiantes, para así contribuir a hacerlas más fuertes y de largo aliento, lo cual será posible si se extienden sus dinámicas hacia las comunidades para armar redes de aprendizaje.

Cabe destacar que todas las experiencias identificadas y las que sabemos se están desarrollando de manera silenciosa en la ciudad y el país, contienen potencialidades inimaginables ante el uso creativo de las tecnologías de la información y la comunicación; las cuales están sobrepasando cada vez más su instrumentalidad, y se dirigen hacia el fortalecimiento de los capitales culturales de las comunidades por la democratización del conocimiento que están propiciando, para hacer, saber, socializar, comunicar y obrar de acuerdo a la problematización de sus contextos más próximos, en el aula y fuera de ella.
Sin embargo, no se puede desconocer la necesidad de un acompañamiento en cuanto a procesos de sistematización, discusión y elaboración pedagógica y conceptual alrededor de las tecnologías tradicionales y las digitales, con una orientación política, estética y cultural, lo cual puede animar su sostenibilidad y alentar otra serie de búsquedas, preguntas y alcances.

Desde esta perspectiva, el reconocimiento de los saberes tecnomediados en niños, niñas, jóvenes y maestros, se constituye como una oportunidad para seguir indagando en un sentido amplio por las potencialidades de las tecnologías, pues este es el contexto para evidenciar los cruces entre los procesos de diseño de artefactos, el conocimiento que allí se solapa y la apropiación de los dispositivos digitales, todos dispuestos a la generación de mediaciones pedagógicas y comunicativas, para acceder al mundo de la ciencia, el arte y la cultura, las cuales han de promover la formación de niños, niñas, jóvenes y maestros, como sujetos más solidarios e incluyentes.

\section{Referencias}

Buckingham, D. (2002). Crecer en la era de los medios electrónicos. Madrid: Ediciones Morata.

Buckingham, D. (2008). Más allá de la tecnología. Aprendiraje infantil en la era de la cultura digital. Buenos Aires: Manantial.

Martín-Barbero, J. (1999, Octubre). La educación en el ecosistema comunicativo. Comunicar, No. 13, 13-21.
Nussbaum, M. (2012). Crear capacidades. Propuesta para el desarrollo humano. Barcelona: Espasa.

Rueda, R. (2012, Enero-Abril). Educación y cibercultura en clave subjetiva: retos para pensar la escuela hoy. Revista Educación y Pedagogía. Educación y cibercultura: campos de estudio, retos y perspectivas. Medellín: Universidad de Antioquia, Facultad de Educación, Vol. 24, No. 62, 157-171. 
Tema: Nuevos Lenguajes y Educación 